\title{
An Inductively Coupled Plasma-Time-of-Flight Mass Spectrometer for Elemental Analysis. Part I: Optimization and Characteristics
}

\author{
D. P. Myers, G. Li, P. Yang,* and G. M. Hieftje \\ Department of Chemistry, Indiana University, Bloomington, Indiana, USA
}

\begin{abstract}
An inductively coupled plasma-time-of-flight mass spectrometer (ICP-TOFMS) has been constructed and evaluated for elemental analysis. The instrument produces analog spectra similar to those from quadrupole inductively coupled plasma mass spectrometers. The large abundance of Ar ions is deflected away from the microchannel plate detector to reduce detector dead time and space-charge complications. The ICP-TOFMS, operated in a linear (nonreflecting) mode, currently has a resolving power of 500 (full width at half maximum). Present ion optics employed in the instrument require a trade-off between signal-to-noise ratio and resolving power. In addition, mass-dependent kinetic energies in the supersonic beam created in the ICP mass spectrometer interface cause a mass bias in the right-angle TOFMS because the ions must be steered to the detector to compensate for their velocity in the supersonic beam direction. In the current design the sampling duty cycle is only approximately $3 \%$, thereby limiting sensitivity. However, positive potentials applied to the right-angle extraction region can increase sensitivity by a factor of 2-4 by slowing down the ions that enter the extraction zone. The transmission efficiency of the TOFMS is approximately $20 \%$ and is limited by divergence of the ion packet in the drift tube. (J Am Soc Mass Spectrom 1994, 5, 1008-1016)
\end{abstract}

$\mathrm{R}$ cently a renewed interest has emerged in atmospheric-pressure ionization (API) time-of-flight, (TOF) mass spectrometers, Yet, coupling an ion source at atmospheric pressure to a time-of-flight mass spectrometer (TOFMS) is not straightforward and must be tailored to a chosen ion source. One of the first TOFMS instruments to 11se an atmospheric-pressure ion source was described in the mid-1960s by workers at the Bendix Corporation [1]. They built an orthogonal TOFMS that was coupled to a plasma ion source. Several other groups more recently involved in APITOFMS also have employed orthogonal ion acceleration in the TOF analyzer [2-5], although others have adopted an axial geometry [6]. Ion reflectors have been employed for both axial and orthogonal arrangements $[2,6]$.

Inductively coupled plasma mass spectrometry (ICP-MS) is a powerful method of trace multielement analysis [7]. Ordinarily, quadrupole or double-focusing instruments have been utilized for ICP-MS. However, we recently coupled a time-of-flight mass spectrometer to an inductively coupled plasma (ICP) ion source [8] to assess its performance. The primary advantage of

* Current address: Department of Chemistry, Xiamen University, Xiamen, FJ 361005, People's Republic of China,

Address reprint requests to Dr. Gary $M$. Hieftje, Department of Chemistry, Indiana University, Bloomington, IN 47405. the TOFMS is its ability to produce a spectrum of all the ions it samples in a very short period of time, in the range of $30-100 \mu \mathrm{s}$ for all the chemical elements. In addition, for appropriate designs of TOFMSs [9] the ion transmission can be very high $(>50 \%)$. Furthermore, because elemental isotopes would be detected very close together in time (nanoseconds), and because all the ions in a single spectrum would be extracted simultaneously from the ICP, the measurement uncertainties in isotope-ratio measurements compared to scanning mass spectrometers may be lower [10]. In fact, isotope-ratio measurements with resonance ionization TOFMS and the laser microprobe mass analyzer have shown considerable promise [10-12]. The speed of the TOFMS could also improve the analysis of transient samples in ICP-MS.

The studies reported here will focus on the modification and optimization of the ion optical and ion sampling system in an orthogonal TOFMS to improve ion transmission efficiency and consequently the instrumental sensitivity. A primary concern in the development of this instrument is how best to pulse a continuous ion beam into the TOFMS to achieve a high sampling duty factor. Because only a short burst of the ions in the continuous beam generated by the ICP is needed for TOFMS analysis, the choice of an appropriate sampling of the beam is the most important factor to insure adequate sensitivity in the TOFMS. Unfortunately, as we will show, use of an orthogonal sampling 
geometry to increase the duty cycle may lead to poorer ion transmission in the TOFMS.

For gaseous ion sources in vacuum, the resolving power of linear Wiley-McClaren [13] types of TOFMSs is historically between 500-1000. Fortunately, orthogonal sampling and proper space focusing can alleviate possible resolution difficulties with a continuous atmospheric-pressure ion source. The attainable fesolving power appears to be adequate for elemental analysis.

\section{Experimental}

\section{Ion Source}

$\Lambda \mathrm{n}$ argon inductively coupled plasma (ICP) at atmo spheric pressure serves as the source of elemental ions. The plasma is maintained by a power supply operating at $40 \mathrm{MHz}$ (Plasma-Therm, Kresson, NJ). A commercial tangential-flow quartz torch (Precision Glass Blowing, Englewood, $\mathrm{CO}$ ) is used to support the plasma. Aqueous solutions are introduced into the central channel of the ICP with (1) a locally constructed ultrasonic nebulizer [14] followed by desolvation or (2) a glass concentric nebulizer and Scolt-type spray chamber with no desolvation. The plasma is sampled through a $0.75-$ or $1-\mathrm{mm}$ orifice in the sam- pling plate of the mass spectrometer at $10 \mathrm{~mm}$ above the load coil. Table 1 contains a summary of the operating conditions.

\section{Mass Spectrometer}

Many of the components used in this instrument were described earlier [8]. Here the greatest emphasis will be placed on portions that have been modified. The TOFMS is a modified angular reflectron constructed by R. M. Jordan Co. (Grass Valley, CA). The inductively coupled plasma is interfaced to the TOFMS via a conventional three-stage differentially pumped interface. The entrance to the first stage of the vacuum system is a $0.75 \mathrm{~mm}$ (or $1-\mathrm{mm}$ ) orifice in a water-cooled copper sampling plate. Located $10 \mathrm{~mm}$ downstream is a $1-\mathrm{mm}$ (or $0.5-\mathrm{mm}$ ) skimmer orifice. With a $0.75-\mathrm{mm}$ sampling orifice and $1-\mathrm{mm}$ skimmer orifice the TOFMS base pressure is $1 \times 10^{-5}$ torr. The ion optical configuration is more unstable at these higher pressures; therefore, studies at lower pressures were performed by increasing the first-stage orifice to $1 \mathrm{~mm}$ and decreasing the skimmer orifice to $0.5 \mathrm{~mm}$. These orifices result in a third-slage pressure of $5.5 \times 10^{-6}$ torr (see Table 1).

The entire instrument is shown in Figure 1. The ion

Table 1. ICP-TOFMS operating conditions

\begin{tabular}{|c|c|c|}
\hline & For Figures $3-6,8,10$ & For Figures $12-14$ \\
\hline $\begin{array}{l}\text { Sample } \\
\text { introduction }\end{array}$ & $\begin{array}{l}\text { Ultrasonic nebulizer with } \\
\text { desolvation }\end{array}$ & $\begin{array}{l}\text { Class concentric nebulizer, } \\
\text { no desolvation }\end{array}$ \\
\hline Plasma power & $1.35 \mathrm{~kW}$ (<5W reflected $)$ & $1.35 \mathrm{~kW}$ (<5 W reflected $)$ \\
\hline Central gas flow & 1.1 to $1.4 \mathrm{~L} / \mathrm{min}$ & 1.2 to $1.3 \mathrm{~L} / \mathrm{min}$ \\
\hline Intermediate flow & $1.0 \mathrm{~L} / \mathrm{min}$ & $1.0 \mathrm{~L} / \mathrm{min}$ \\
\hline Outer gas flow & $13.0 \mathrm{~L} / \mathrm{min}$ & $13.0 \mathrm{~L} / \mathrm{min}$ \\
\hline Sampling position & $10 \mathrm{~mm}$ above load coil & $10 \mathrm{~mm}$ above load coil \\
\hline Sampling orifice diameter & $0.75 \mathrm{~mm}$ & $1.0 \mathrm{~mm}$ \\
\hline Skimmer orifice diameter & $1.0 \mathrm{~mm}$ & $0.5 \mathrm{~mm}$ \\
\hline First-stage pressure & 0.90 torr & 1.07 torr \\
\hline Second-stage pressure & $1 \times 10^{-3}$ torr & $4.8 \times 10^{-4}$ torr \\
\hline Third-stage pressure & $1 \times 10^{-5}$ torr & $5.5 \times 10^{-6}$ torr \\
\hline \multicolumn{3}{|c|}{ Ion optic voltages (see Figure 1) } \\
\hline$\$ 1$ & $-800 V$ & $-800 \mathrm{~V}$ \\
\hline L1 & $-650 \mathrm{~V}$ & $-620 \mathrm{~V}$ to $-720 \mathrm{~V}$ \\
\hline L2 & $-100 v$ to $-200 v$ & $-100 \mathrm{~V}$ to $-200 \mathrm{~V}$ \\
\hline L3 & $-20 \mathrm{~V}$ to $-30 \mathrm{~V}$ & $-200 \mathrm{~V}$ to $-220 \mathrm{~V}$ \\
\hline R bias potential & 0 to $-2 V$ & -6 to $11 \mathrm{~V}$ \\
\hline R pulse potential & 147V@10kHz & 147 to $171 \mathrm{v} @ 10$ kHz \\
\hline G1 & ground & -2 to $15 \mathrm{~V}$ \\
\hline $\mathrm{G} 2$ & $-2000 \mathrm{~V}$ & $2000 \mathrm{~V}$ \\
\hline D1 bias potential & $-1966 V$ & $-1966 \mathrm{~V}$ \\
\hline D1 pulse potential & $-200 \mathrm{~V} @ 10 \mathrm{kHz}$ & $-200 \mathrm{~V} @ 10 \mathrm{kHz}$ \\
\hline D1 pulse width & $310 \mathrm{~ns}$ & $310 \mathrm{~ns}$ \\
\hline D1 pulse delay & $4.00 \mu \mathrm{s}$ & $4.00 \mu \mathrm{s}$ \\
\hline \multicolumn{3}{|l|}{ Detector voltages } \\
\hline MCP front & $-2880 \mathrm{~V}$ & $-2880 V$ \\
\hline MCP center & $-1920 \mathrm{~V}$ & $-1920 V$ \\
\hline MCP back & $-960 v$ & $-960 \mathrm{~V}$ \\
\hline
\end{tabular}


optics in Figure 1 consist of a second-stage extraction element (S1) that contains a $1.3-\mathrm{mm}$ conductionlimiting orifice positioned 15 mun behind the skimmer cone. Behind that orifice, in the third stage of vacuum, lie three more lens elements (L1, L2, L3). The thirdstage lenses further focus ions into the region between the repeller (R) and the first entrance grid (G1) of the TOFMS (Figure 1). The last element (L3) has a rectangular exit slit, the long axis of which is perpendicular to the TOF flight tube and is $2.5-\mathrm{mm}$ wide. This slit width offers improved resolving power, sensitivity, and noise levels. The first entrance grid (G1) is ordinarily maintained at ground potential, whereas the repeller potential is adjusted to be slightly negative (1-3 v) for maximum total ion currents and low scatteredion noise. The application of positive potentials to both the repeller and the first entrance grid will be studied in experiments to be discussed later. The total ion current in the primary beam is measured with a Faraday cup (C) at the exit of the region defined by the repeller and the first grid.

The TOFMS uses a pulsed extraction, applied at a controllable repetition rate of $5-10 \mathrm{kHz}$, followed by an acceleration zone. The pulsed extraction is accomplished by applying a positive pulse to the repeller, which sends ions present in the continuous beam into the second step of acceleration. This second step consists of a linear static field maintained on four rings (2.54-cm diameter) and a second entrance grid (G2), also 2.54-cm diameter. Grid G2 is biased at $-2000 \mathrm{~V}$ and equal resistance between the ring elements produce a linear potential gradient across the second acceleration region. This second region is approximately five times the distance between the repeller and the first entrance grid (G1), which proved to yield optimal resolving power. The potential applied to $\mathbf{G} 2$ is also applied to the drift tube, which is insulated from the vacuum chamber walls. Steering plates $(2.54 \mathrm{~cm}$ long and separated by $2.54 \mathrm{~cm}$ ) are located after the second entrance grid (G2) to correct for the initial perpendicu-

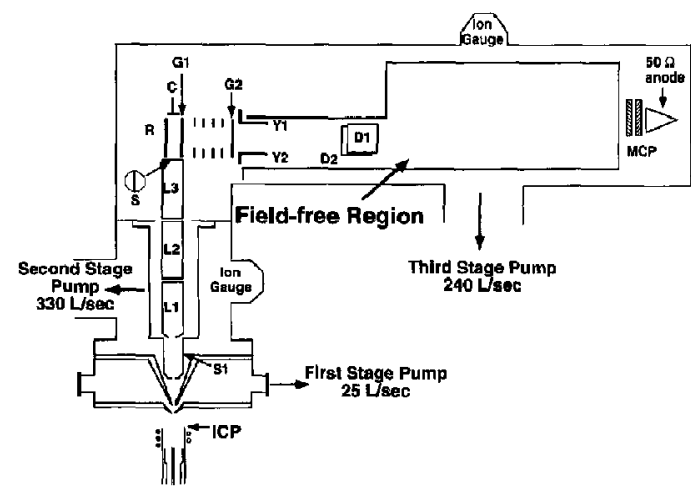

Figure 1. Diagram of ICP-TOFMS. $S 1$ is the second-stage extraction lens, L1, L2, and $\mathrm{L} 3$ are third-stage optics, $\mathrm{S}$ is the $2.5-\mathrm{mm}$ slit inside exit of the L3 optic, $\mathrm{R}$ is the repeller, $\mathrm{C}$ is the Faraday cup, G1 and G2 are the TOFMS entry grids, $Y 1$ and $Y 2$ are the steering plates, D1 and D2 are the deflection plates, and MCP is the dual chevron microchannel plates. lar velocity component of the supersonic beam. Plate $\mathrm{Y} 1$ is maintained at $-2000 \mathrm{~V}$, whereas $\mathrm{Y} 2$ is tuned mure negatively to direct ions to the detector (MCP). These steering plates are orthogonal to the direction of the original supersonic beam.

Located $30 \mathrm{~cm}$ from the center of the right-angle extraction region (defined by $R$ and G1) are two deflection plates $(3.8 \mathrm{~cm}$ long and separated by $3.2 \mathrm{~cm}$ ), one of which is maintained at the flight tube potential. The second plate is held close to the flight tube potential, but receives a $-200-\mathrm{V}$ pulse at an adjustable delay time after the repeller pulse. This delay and pulse width are adjusted to minimize the $\mathrm{Ar}^{+}$signal at the detector. These deflection plates are perpendicular to the steering plates (see Figure 1). The detector is located $114 \mathrm{~cm}$ from the center of the right-angle extraction region and is a $23-\mathrm{mm}$ dual chevron microchannel plate (MCP) assembly (Galileo Electrooptics Corp., Sturbridge, MA).

\section{Detection Electronics}

The output of the chevron MCP is sent to a biasing circuit to adjust the baseline of the spectra to be as close as possible to $0.0 \mathrm{~V}$. The signals are then sent to a wide-band amplifier (20 dB. CLC140, Comlinear, Fort Collins, CO) and dc-coupled into the input of a Tektronix (Beaverton, OR) TDS 520 digital oscilloscope (500 Msamples/s) and collected via a GPIB interface (National Instruments, Austin, TX) on a Macintosh Ouadra 950 computer.

\section{Results and Discussion}

\section{Background Spectra}

Background spectra obtained on the ICP-TOFMS are similar to those from quadrupole ICP-MS systems. Figure 2 shows the background ions over the 1-50-u range (distilled deionized $\mathrm{H}_{2} \mathrm{O}$ ) as the oscilloscope displays them. Because the $\mathrm{Ar}^{+}$is selectively deflected during this time-of-flight mass analysis, the peak seen at $40 \mathrm{u}$ is diminished and the shape is affected ad-

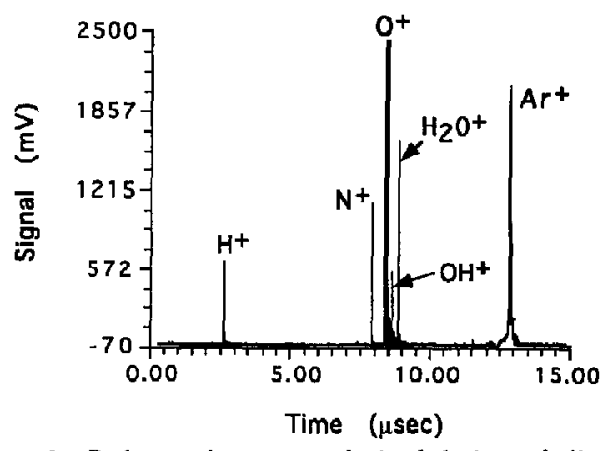

Figure 2. Background spectrum obtained during nebulization of distilled deionized water (average of 1000 shots). 
versely. However, the $\mathrm{O}^{+}$peak still saturates the preamplifier (maximum specified output is $1.5 \mathrm{~V}$ ). In addition, the removal of masses close to $\mathrm{Ar}^{+}$is unavoidable due to the less than optimal location and dimensions of the deflection plates and the width of the deflection pulse ( $310 \mathrm{~ns})$. Masses at $39 \mathfrak{u}$ (e.g., ${ }^{39} \mathrm{~K}$ ) are currently impossible to measure at low concentrations because of the argon peak.

The effect of the $\mathrm{Ar}^{+}$deflection pulse width can be seen in Figure 3, where spectra of a 20-ppm solution of $\mathrm{Sc}$ and $\mathrm{K}$ are shown. The longer pulse width (Figure 3a) is obviously more effective at removing $\mathrm{Ar}^{+}$; however, masses near $\mathrm{Ar}^{+}$(e.g., $\mathrm{ArH}^{+}$) are also removed. Reducing the deflection pulse width to 138 ns (Figure $3 \mathrm{~b}$ ) allows the $\mathrm{ArH}$ ' ions to hit the detector, but the $\mathrm{Ar}^{+}$ion begins to saturate it. In the future, we intend to relocate the deflection plates at a space focus plane [9] to remove $\mathrm{Ar}^{+}$ions more effectively.

\section{Resolving Power}

The use of a two-step acceleration field with pulsed extraction allows the repeller pulse amplitude (first step) to be adjusted for the best first-order space focusing [13] at the detector of the TOFMS. We have found also that the width of the slit in the last lens element dictates the attainable resolving power. This resolving power can be seen in Figure 4 for light and heavy ions.
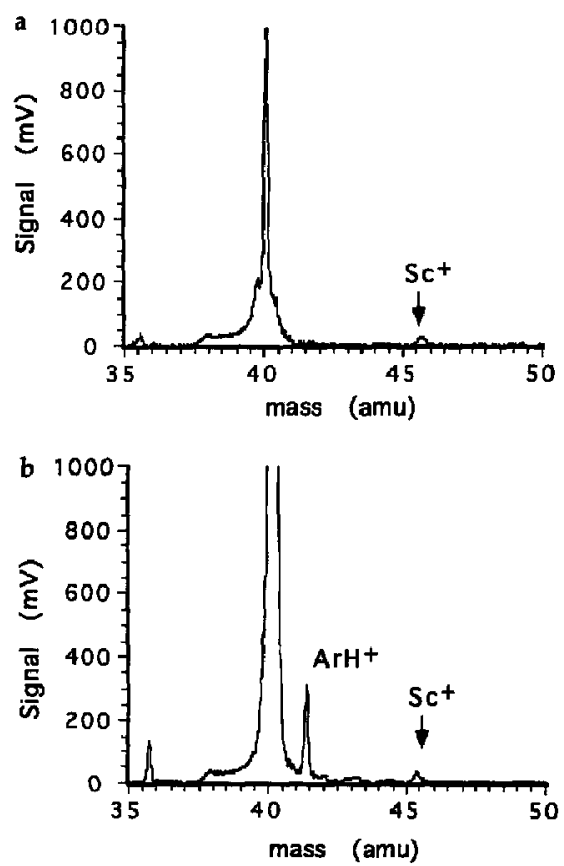

Figure 3. Spectra (average of 1000 shots) of 35-50-u mass range for differing $\mathrm{Ar}^{+}$deflection pulse widths (both spectra are for 20 -ppm Sc and K). (a) width $=310 \mathrm{rts}$, delay from sample-inptit pulse $=4.00 \mu \mathrm{s}$; (b) width $=138 \mathrm{~ns}$, delay $=4.00 \mu \mathrm{s}$.
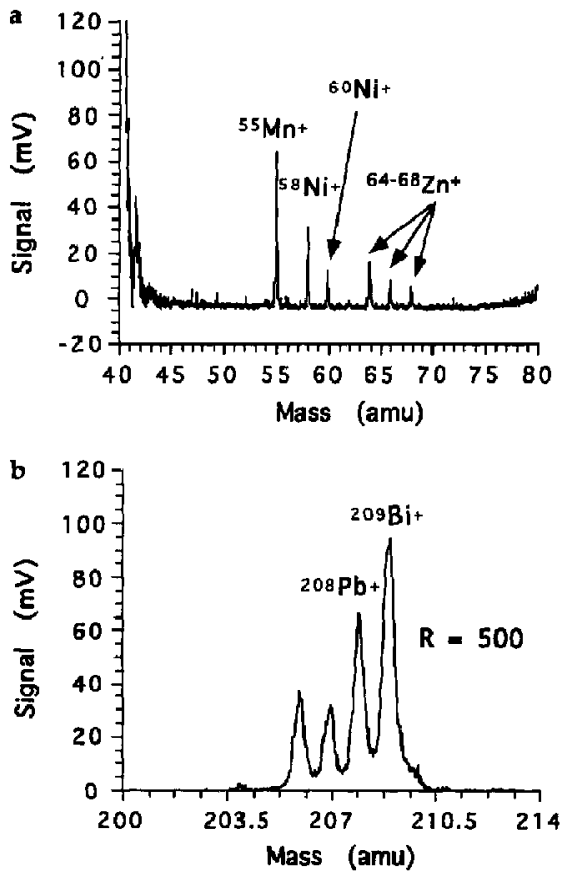

Figure 4. Spectra (average of 1000 shots) for mass range from (a) 40-80 u and (b) 200-214 u. Both spectra are for 20-ppm Mn, $\mathrm{Ni}, \mathrm{Zn}, \mathrm{Pb}$, and $\mathrm{Bi}$.

After optimization of the center element of the thirdstage ion optics (L2 in Figure 1), a resolution [full width at half maximum (FWHM)] of $500 \mathrm{can}$ be obtained for a $\mathrm{Pb}-\mathrm{Bi}$ solution (Figure $4 \mathrm{~b}$ ). Notice also that in Figure $4 \mathrm{~b}$ the overall ratio of total $\mathrm{Pb}$ to $\mathrm{Bi}$ seems high for a solution of equal concentration. The only plausible explanation is that the concentration of $B i$ is actually less than expected. We would not expect any mass discrimination between ions so close in mass, as will be described later. The resolving power is promising; however, the only difficulty might arise when a very abundant ion is next to a trace ion. That is, for acceptable abundance sensitivity the resolving power might require improvement. This improvement could be realized in several ways. The ion optic lenses in the third stage could be designed to focus the ion beam into a slit-like image for orthogonal extraction into the TOFMS. Such an image would have less ionbeam divergence in the extraction zone and should offer better first-order space focusing and, therefore, better resolving power. In addition, the use of an ion reflector for velocity focusing will certainly improve resolution.

\section{Ion Optics (Signal-to-Noise Ratio and Resolution)}

As mentioned above, efficient extraction and focusing of ions from the ICP is the most important design aspect of the ICP-TOFMS. The optics employed in the 
ICP-TOFMS extract ions from the supersonic beam through a cylindrical optic (S1; see Figure 1) with a 1.3-mm urifice that is located in the second stage of differential pumping at a point $15 \mathrm{~mm}$ behind the skimmer. Inside S1 the pressure is approximately $10^{-5}$ torr; thus, the ions travel only a short distance in the second stage of the vacuum system (roughly $10^{-3}$ torr). Optimization studies showed that voltages on S1 less negative than about $-500 \mathrm{~V}$ yield low total ion currents (measured at $C_{\text {; }}$ see Figure 1) and TOFMS signals, whereas potentials more negative than $-800 \mathrm{~V}$ do not significantly improve currents or signals. In the third stage of vacuum, beyond $S 1$, a three-element lens (L1, L2, L3) focuses ions into the mass spectrometer entrance (the extraction region). The first element (L1) of the lens arrangement is quite sensitive and optimizes at $-650 \mathrm{~V}$. The last element, L3, has a minimal effect on resolving power and influences mainly the ion signal level. Consequently, L3 is optimized at a potential that yields the highest signal-to-noise $(\mathrm{S} / \mathrm{N})$ ratio.

Resolution and $\mathrm{S} / \mathrm{N}$ are affected most strongly by the potential applied to the center lens element (L2). A sacrifice in $\mathrm{S} / \mathrm{N}$ ratio is necessary to achieve the resolution shown in Figure 4a. Figure 5 shows quantitatively how $\mathrm{L} 2$ affects $\mathrm{S} / \mathrm{N}$ and resolution. This trade-off of $S / N$ for resolving power is a result of the ion optical setup. Figure 6 shows a Maçimion ${ }^{\text {(8) }}$ (version 2.0, Montech Pty. Ltd., Australia) trajectory calculation for the ion optics comprised of the skimmer, S1, L1, L2, and L3. Because the geometry of the lens system is cylindrical, the $2.5-\mathrm{mm}$ slit in lens L3 could not be included in the trajectory calculations. However, to show the problem with the lens system we have added the exit slit to the trajectory diagrams. The trajectories for an $L 2$ potential that yields maximum $S / N$ ratio (Figure 6a) shows that the focal point is closer to the exit slit; therefore, transmission through the slit is high and more ions can enter the extraction zone. One would expect that the maximum signal would correspond to the situation where the ion beam is focused

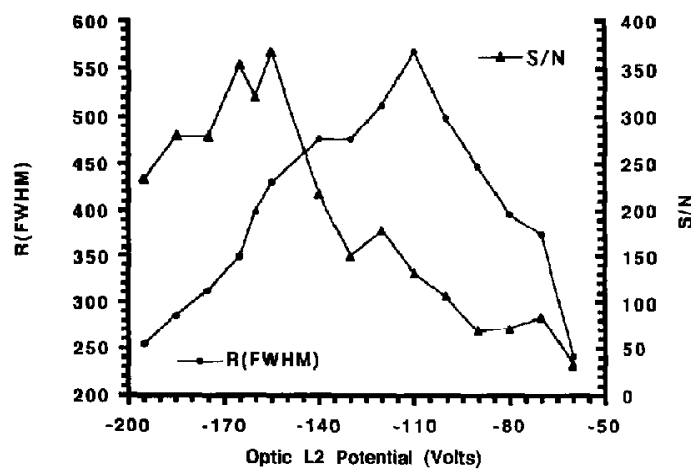

Figure 5. Effect of optic L2 potential on $\mathrm{S} / \mathrm{N}$ ratio and resolution $(R)$ for 20-ppm $\mathrm{Pb}$.
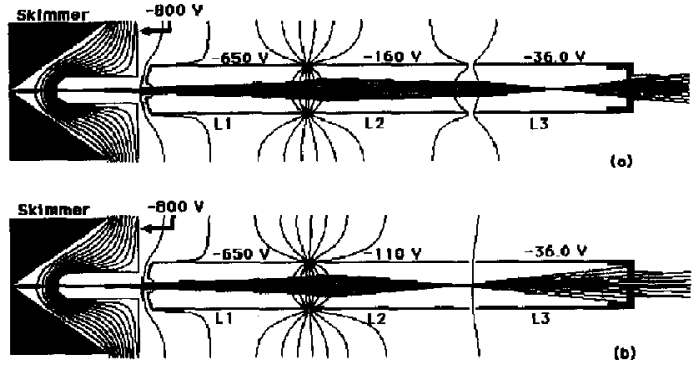

Figure 6. MacSimion trajectories for $\mathrm{Ar}^{+}$(initial energy $10 \mathrm{eV}$; 10 initial angles shown at $2^{\circ}$ intervals from -10 to $+10^{\circ}$ ) at optic $\mathrm{L} 2$ potentials that yield maximum $S / N$ ratio (a) $L 2=-160 \mathrm{~V}$ and resolution (b) $\mathrm{L} 2=-110 \mathrm{~V}$.

on the slit (higher L2 potentials). However, Figure 5 shows that the $\mathrm{S} / \mathrm{N}$ falls off at these higher potentials. The main reason $\mathrm{S} / \mathrm{N}$ falls off in this region is due to the fact that more ions enter the extraction zone during the time when the repeller pulse is off, and these ions create more noise at the detector. In addition, in Figure 6a the ion beam then diverges greatly after it passes the slit, worsening space focus and degrading resolution. In contrast, trajectories for conditions that yield maximum resolving power (Figure 6b) show that the focal point is farther from the exit slit. Although fewer ions enter the extraction zone, those that are transmitted diverge less than those shown in Figure 6a. The resulting planar beam of ions can be brought to a better first-order space focus at the TOFMS detector, so resolving power is improved. These simulations suggest that an ion-optical system that forms a rectangular image and nearly parallel ion beam in the right-angle region would obviate the need for this trade-off between $\mathrm{S} / \mathrm{N}$ ratio and resolution. Of course, the simulations must be weighed cautiously because spacecharge effects in the ion beam were not considered.

\section{Ion Noise}

In single-shot spectra obtained on the ICP-TOFMS, noise from random ion hits can be seen across the entire flight-time domain (Figure 7). Presumably this "ion noise" is caused by the continuous unintentional injection of ions into the TOFMS. Ion packets from the continuous ion beam are extracted in 2- $\mu$ s time periods at a repetition rate of $10 \mathrm{kHz}$. Throughout the duration of the repeller pulse no ions can enter the extraction zone because of the electric field that is present. However, when the pulsed ions are being mass analyzed, ions in the supersonic beam are able to fill up the extraction volume. The divergent ion beam in this region (see Figure 6) permits these unwanted ions to enter the flight region at all times. The ions that enter have roughly $5-20 \mathrm{eV}$ of kinetic energy; a diver- 


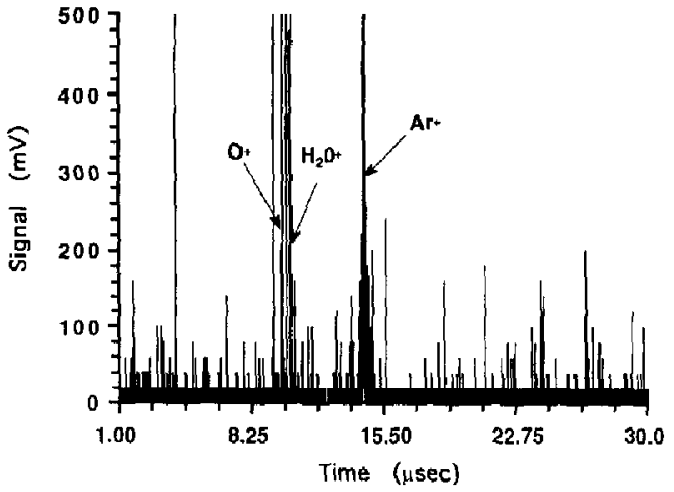

Figure 7. Single-shot spectrum obtained from distilled deionized water that shows background ion noise.

gent trajectory in the direction of grid G1 would send them into the acceleration region. Support for this "ion noise" hypothesis is obtained by applying a small negative potential to the repeller plate. This action reduces the ion noise tremendously; however, resolving power and $S / \mathrm{N}$ are adversely affected. As will be discussed later, ion noise can be reduced by other means.

\section{Mass-Dependent Energies and Steering Plate Bias}

In accordance with the expected behavior of a supersonic beam, ions in the continuous beam extracted from the ICP all move with the same velocity. As a result, ions possess a kinetic energy proportional to their mass. This spread in kinetic energies remains, despite the focusing effects of lens elements S1, L1, L2, and L3. This kinetic-energy spread causes ions of different mass to follow disparate trajectories when they are deflected into the perpendicular flight tube. Example trajectories are shown for three masses in Figure 8. Of course, the velocities of the three masses $\left(v_{y 1}, v_{y 2}\right.$, $\left.v_{y 3}\right)$ in the supersonic beam are equal. Mass $m_{1}$ is lighter than $m_{2}$, which is lighter than $m_{3} ;$ therefore, the velacities along the flight tube axis satisfy the

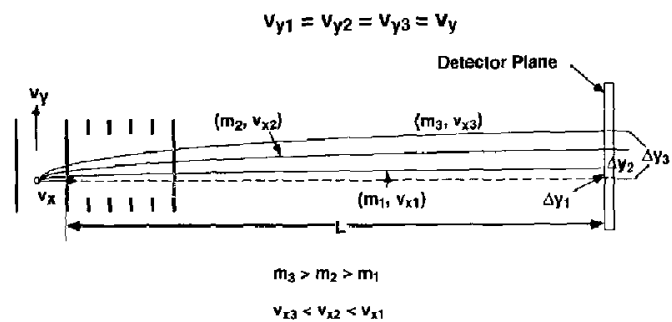

Figure 8. Example trajectories and displacement on the detector plane for ions of three masses $\left(m_{1}, m_{2}, m_{3}\right), v_{41}, v_{42}$, and $v_{43}$ are velocities of the ions in the supersonic beam; $v_{x 1}, v_{x 2}$, and $v_{x 3}$ are velocities of the ions along the flight tube axis $\Delta y_{1}, \Delta y_{2}$, and $\Delta y_{3}$ are displacements of the ions in the detector plane; $L$ is the flight length. inequality $v_{x 3}<v_{x 2}<v_{x 1}$, which is essential for mass analysis in a drift length $L$. The heavier masses have a lower velocity in the flight tube direction and more energy in the supersonic beam, and therefore are displaced farther in the detector plane. The displacements of the lightest to heaviest ions are represented by $\Delta y$ in Figure 8. Other researchers have reported similar effects in a right-angle TOFMS $[1,5,12]$.

To promote ion trajectories that end at the detector surface, ions must be steered with an energy field in the flight region; steering plates $Y 1$ and $Y 2$ (see Figure 1) accomplish this task. However, the mass-dependent spread in ion kinetic energies in the original beam requires different steering fields for ions of different masses. This requirement is illustrated in Figure 9, which shows the effect of the steering field on ion signals over the elemental mass range. Clearly mass discrimination exists and will pose a problem if ions over a large mass range (such as $\mathrm{Li}$ and $\mathrm{Bi}$ ) must be determined simultaneously. However an intermediate field can be chosen as a compromise.

The large range of steering fields that produce detectable signals suggests that the ion beam in the flight region diverges substantially. This divergence certainly lowers ion transmission and raises noise from scattered ions. Furthermore, although the steering plates can compensate for the kinetic energies in the supersonic beam, fringing fields associated with plates of this length likely degrade resolving power [15].

\section{Duty Cycle / Transmission Efficiency}

One of the reasons to choose an orthogonal ICP-TOFMS is to increase the duty cycle of the mass spectrometer $[2-5,8]$. A TOFMS can accept an input pulse of ions only after the previous input ion packet is mass analyzed. Consequently, its duty cycle is usually rather poor, and can yield poor sensitivity compared to continuous ion-beam mass spectrometers. However the right-angle TOFMS takes advantage of the fact that

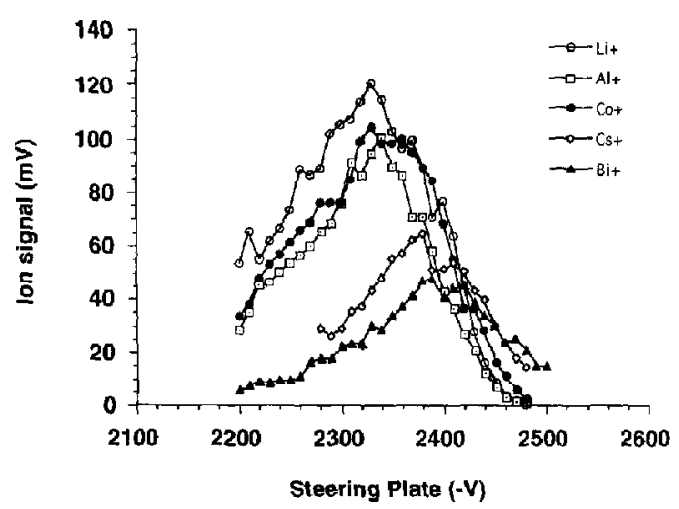

Figure 9. Ion signals versus steering potential (Y2) for ions of differing mass. 
ions in the initial supersonic beam move at velocities typically 1-2 orders of magnitude lower than those in the flight tube. As a result, the extraction region can be filling slowly while the previous extracted pulse is being analyzed. The duty cycle of such an instrument can be calculated from the following equation:

$$
\text { duty cycle }=(b)(f) / v
$$

where $b$ is the length of the extraction zone, $f$ is the frequency at which input ion pulses are extracted, and $v$ is the initial beam velocity in the direction orthogonal to the drift tube. By using the parameters of our specific ICP-TOFMS - $b=25 \mathrm{~mm}, f=10 \mathrm{kHz}$, and $v \sim 7.1 \times 10^{3} \mathrm{~m} / \mathrm{s}$ (corresponding to Ar)-we calculate the duty cycle to be only $3.5 \%$.

Obviously it would be desirable to raise this value. Unfortunately, the pulsing frequency cannot be increased readily because of electronic constraints; high pulse repetition rates translate into higher average power. In addition, pulsing at a higher frequency would require shorter flight times which would demand a higher accelerating voltage and, in turn, a greater extraction-pulse amplitude to maintain space focusing.

Two more attractive options to improve the duty factor are (1) to increase $b$, the extraction length, or (2) to reduce the ion velocity in the primary beam. Increasing the length of the extraction zone is not attractive because the beam must not become larger than a typical MCP detector (23-40-mm diameter). Furthermore, transmission efficiency is poorer for a lengthened ion packet. Thus, lowering the ion velocity in the primary beam seems most feasible. The difficulty of transmitting a lengthened ion packet through the ICPTOFMS should also be considered.

Ion throughput in the TOFMS can be raised not only by means of a greater duty factor, but also by increasing the TOFMS transmission directly. By comparing theoretical transmission with measured values in the ICP-TOFMS we can draw conclusions about the overall efficiency of the mass analyzer. In theory, the transmission efficiency of the orthogonal ICP-TOFMS is limited because of the divergence of such a wide ion packet $(25 \mathrm{~mm})$ in the drift length. The divergence of the extracted ions can be estimated, thus yielding an approximate percentage of ions that hit the detector surface. In Figure 10 the divergence of the ion beam is represented. The extracted length in our TOFMS is 25 $\mathrm{mm}$ and the detector diameter is $23 \mathrm{~mm}$. The angle of divergence $\alpha$ can be estimated as follows (see Figure 10):

$$
\tan \alpha=v_{y} / v_{x}
$$

where $v_{y}$ is the velocity spread in the supersonic beam and $v_{x}$ is the ion velocity down the flight tube. For

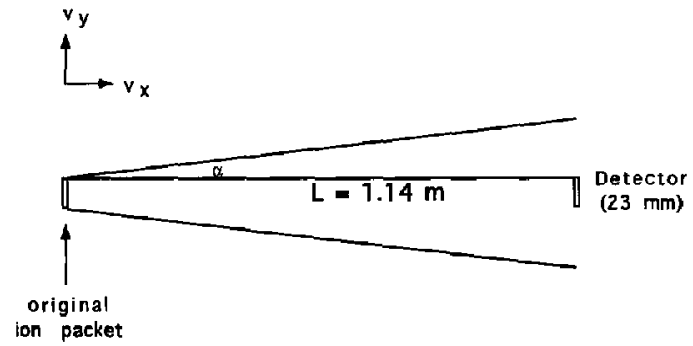

Figure 10. Ion beam divergence in the ICP-TOFM. $\alpha$ is the angle of divergence, $v_{y}$ is the velocity spread in the supersonic beam, and $v_{x}$ is the velocity in the flight tube direction (not to scale).

small angles of divergence $\alpha \sim \tan \alpha$ and

$$
\alpha=v_{y} / v_{x}=\left(\Delta K_{y} / K_{x}\right)^{1 / 2}
$$

where $\Delta K_{y}$ is the energy spread in the direction orthogonal to the flight tube and $K_{x}$ is the acceleration potential of the TOFMS. Using typical values for the ICP-TOFMS $(\Delta K \sim 5 \mathrm{eV}, K=2000 \mathrm{eV}$, so $\alpha=0.05)$ and a flight length of $1.14 \mathrm{~m}$, the original ion packet is calculated to spread to a total length of approximately $125 \mathrm{~mm}$. Therefore, the $\mathrm{MCP}$ detector can intercept only about $20 \%$ of the total divergent area of the ion packet. Consequently, in theory, one would expect no better than a $20 \%$ transmission efficiency for the orthogonal ICP-TOFMS. The preceding theoretical estimates are supported by measurements in the ICP. TOFMS. In Figure $4 b$ the signal-averaged peak for $B i$ is approximately $95 \mathrm{mV}$ in amplitude and has a FWHM of $29 \mathrm{~ns}$. Accounting for the preamplification yields an amplitude of $9.5 \mathrm{mV}$ from the MCP that, for the $50-\Omega$ system, means $1.9 \times 10^{-4} \mathrm{C} / \mathrm{s}(\mathrm{A})$ of anode current. During the 29-ns time period $5.5 \times 10^{-12} \mathrm{C}$ are detected. Because the gain of our MCP is estimated to be $\sim 5 \times 10^{6}$, an ion current of $1.1 \times 10^{-13} \mathrm{C}$ hits the front of the MCP. Therefore, this amount of charge corresponds to an average of approximately seven ions per shot for 20-ppm Bi. Next, the flux of ions that enter the mass spectrometer can be calculated from the current that enters the extraction zone, the extraction length, and the ratio of $\mathrm{Ar}$ ions to analyte ions. The measured $\mathrm{Ar}^{+}$/analyte ion ratio for a solution of 1-ppm analyte is $7 \times 10^{5}$ in our instrument. The total ion current measured at the Faraday cup, repeller, and grid 1 (G1) is approximately $60 \mathrm{nA}$. Thus, a $20-\mathrm{ppm}$ solution of $\mathrm{Bi}$ will produce $\sim 38$ ions in the $2.54-\mathrm{cm}$ extraction length, considering their velocity in the supersonic beam is $7.1 \times 10^{3} \mathrm{~m} / \mathrm{s}$. These rough calculations from measurements in our ICP-TOFMS yield an expected transmission efficiency from the extraction region to detector of $\sim 18 \%$, in close agreement with the preceding theoretical estimates regarding the divergent inn packet.

The foregoing discussion leads to the conclusion that the best way to improve the duty cycle and 
sensitivity of our instrument may be to reduce the velocity of ions that enter the extraction region. This goal can be accomplished by applying a positive potential (just smaller than the kinetic energy of the entering ions) to the extraction zone. In spectra presented earlier (Figures 2-4, 7) the ion-extraction region was given a slight overall negative potential due to the small negative bias on the repeller. To produce a positive potential in the extraction region, positive voltages were applied to the repeller (R) and entrance grid I (G1; see Figure 1), with the repeller slightly less positive, to reduce scattered-ion noise.

Positive potentials on the repeller and $\mathrm{Gl}$ increase the detector signals but require different optimal lens settings than those used at the higher pressure (see Table 1). In particular, the last lens element, L3, must be at a potential around $-200 \mathrm{~V}$ compared to about $-30 \mathrm{~V}$ in the higher pressure case. Observation of ion signals as a function of repeller bias potential helps determine if the duty cycle and characteristics of the instrument can be improved.

Figure 11 shows how $\mathrm{Li}, \mathrm{Cs}$, and Ar signals vary with repeller bias. Each plot was obtained by optimizing the potential difference between the repeller and G1 individually for each element. This optimal differ-
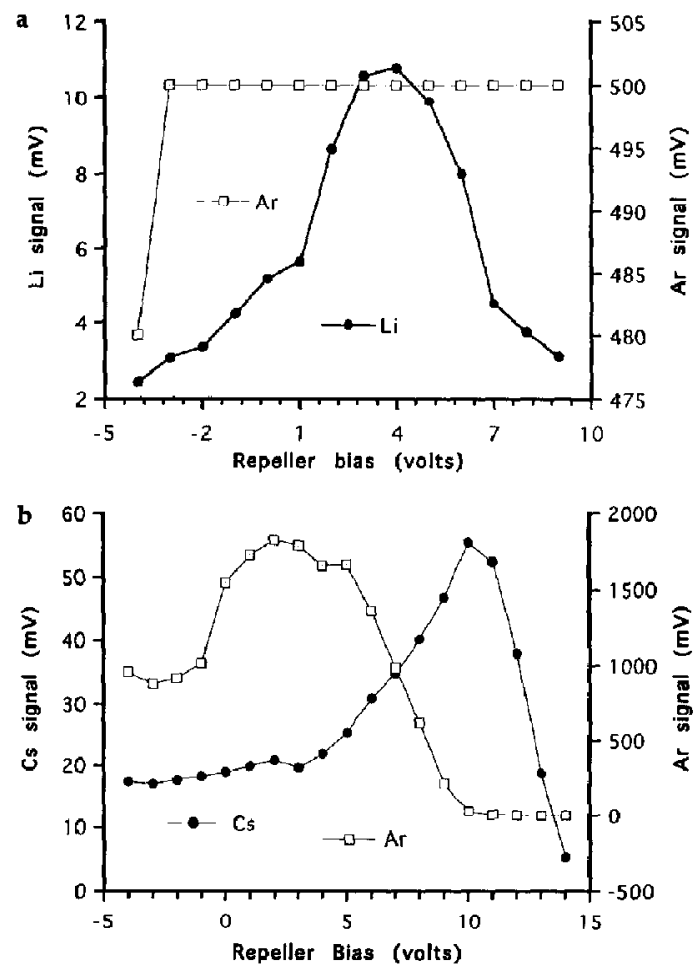

Figure 11. Effect of repeller bias potential on ion signals: (a) 50-ppm $\mathrm{Li}$ and $\mathrm{Ar}$ signals with a $4-\mathrm{V}$ difference between repeller and G1; (b) 50-ppm $\mathrm{Cs}$ and Ar signals with a $7-\mathrm{V}$ difference between repeller and G1. In all cases, G1 is more positive than the repeller by the stated potential difference. ence, listed in the figure captions, was then maintained while the bias (positive) potential was changed. After each bias-potential change the steering-plate voltage (Y2) was readjusted for maximum signal because ion velocity perpendicular to the flight tube was altered. The observation that lithium optimizes at a bias voltage of $4 \mathrm{~V}$ and cesium at a bias of $10 \mathrm{~V}$ supports the fact that $\mathrm{Li}$ has less energy than $\mathrm{Cs}$ in the primary beam. In Figure 11a the Ar signal is unchanged, whereas in Figure 11b the Ar signal declines at higher bias potentials. This behavior is understandable if it is recalled that grid $\mathrm{G} 1$ is $7 \mathrm{~V}$ more positive than the repeller in Figure 11b, but only $4 \mathrm{~V}$ more positive in Figure 11a. What is even more important is that the lighter ions ( $\mathrm{Li}$, here, in Figure 11a or Ar in Figure 11b) can be prevented from entering the TOFMS at high enough repeller bias voltages. This offers a possible scheme for reducing $A{ }^{\prime}$ signals further. This behavior is illustrated further by the composite plot of ion signals versus repeller potential in Figure 12 for five elements from $\mathrm{Li}(m / z=7)$ to $\mathrm{Bi}(m / z=209)$. The increasing ion energy with mass is clearly evident in this plot, as is the overall increase in signals for heavier masses. From Figure 12, the increase in signal attainable with the repeller bias varies from a factor of 3 for lithium to a factor of almost 4 for cesium and bismuth.

Because ions in the primary beam move more slowly with a positive bias applied to the extraction zone, they require a lower steering potential on plate $\mathrm{Y} 2$ (see Figure 1). A plot of the optimal steering field versus repeller bias for the elements in Figure 13 shows both the mass dependence of the field and the lower required steering field as repeller bias is raised (see Figure 13). Of course, a positive repeller bias changes the average ion energies and cannot remove the massdependent effects of the steering plates that were described earlier.

As shown in Figure 12 sensitivity is enhanced less for the lighter elements ( $\mathrm{Li}$ and $\mathrm{Al}$ ) when the positive repeller bias is used, whereas the heavier elements exhibit more enhancement. A possible reason is that the $\mathrm{Li}$ and $\mathrm{Al}$ ions have lower energies than $\mathrm{Ar}$ in the primary ion beam; as a result, the high number density of Ar ions in the extraction zone shields the lighter ions from the positive repeller fields. In contrast, the positive potentials optimal for the heavier elements are high enough to prevent many of the Ar ions from entering the extraction region, which allows the heavier ions to be influenced by the positive repeller potential.

Unfortunately, the use of a positive repeller bias to increase the duty cycle also results in lower resolving power. This observation is not surprising; the spatial distribution of the ion packet in the extraction zone is certainly altered by the positive field there. Apparently this spatial change then reduces the space focusing of the mass spectrometer. Furthermore, resolving power diminishes with mass, which may be a result of fringing fields at the steering plates (heavier ions require a 


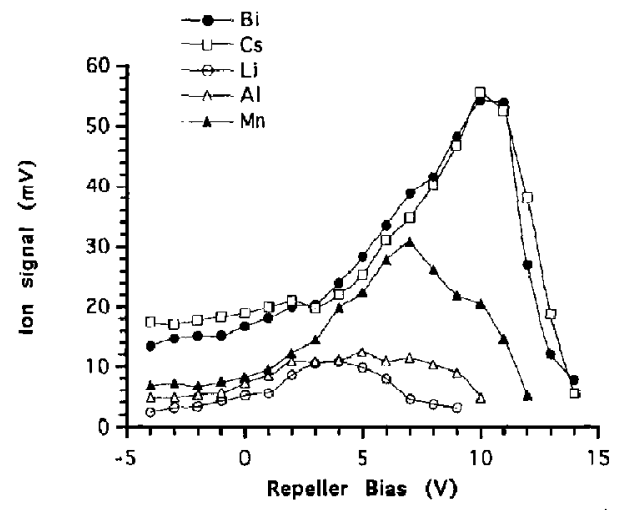

Figure 12. Effect of repeller bias on ion signals from $\mathrm{Li}, \mathrm{Al}$, $\mathrm{Mn}$, Cs, and Bi (all $50 \mathrm{ppm}$ )

larger field to be steered to the detector). Overall, an ion optical system explicitly designed to accommodate the positive potentials in the extraction region may be needed for optimal TOFMS performance.

\section{Conclusions}

An orthogonal ICP-TOFMS has been characterized for use in multielement atomic mass spectrometry. The ion optics used to focus ions into the extraction region are the primary factor in the determination of sensitivity and resolving power. Mass-bias effects exist in the TOFMS because of the mass-dependent ion kinetic energies in the original ion beam. The transmission efficiency of the current TOFMS is no higher than $20 \%$ and the duty cycle is only about $3 \%$. However, positive potentials can be applied to the extraction zone to slow entering ions; the duty cycle increases accord-

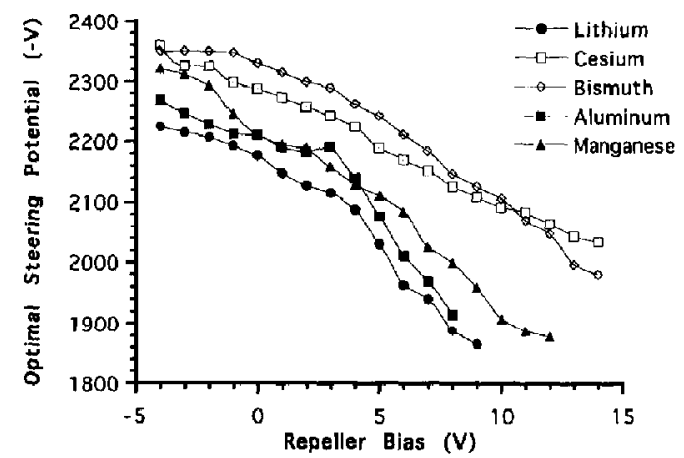

Figure 13. Optimal steering field versus repeller bias. Because the repeller bias potential affects ion energies that enter the extraction region, it similarly influences the optimal stecring bias for those ions to strike the detector. ingly. Currently, the spectral processing system is simply a digital oscilloscope which allows signal averaging. For an averaged spectrum of 1000 shots the real measurement time required is only $0.1 \mathrm{~s}$ at a $10-\mathrm{kHz}$ TOFMS repetition rate. However, the digital scope requires about $40 \mathrm{~s}$ to average 1000 shots; therefore, we also will investigate an improved detection scheme for use in the ICP-TOFMS. Future endeavors will involve the use of an ion reflectron to improve resolving power, modification of the ion optics to eliminate the trade-off between $S / N$ and resolution, and reduction of the mass-bias effects seen in the instrument. The detection system used in the present ICP-TOFMS determines in part the analytical figures of merit; this development will be presented in a later publication.

\section{Acknowledgments}

This research was funded in part by the National Institutes of Health through grant R01 GM 46853. D. P. Myers thanks Eli Lilly $\& \mathrm{CO}_{\text {. }}$, the Department of Education, and the Upjohn Co. for fellowship support.

\section{References}

1. ƠHalloran, G. J.; Walker, L. W. Technical Document Report ASD TDR 62-644, Parts I and II, Nov. 1964. Prepared under Contract AF 33(6.57)-11018 by the Bendix Corporation, Research Laboratories Division, Southfield, MI.

2. Dodonov, A. F.; Chernushevich, I. V.; Laiko, V. V. Book of Abstracts from the 12th International Mass Spectrometry Conference; North-Holland: Amsterdam, 1991; p 153.

3. Coles, I.; Stutsel, M.; Guilhaus, M. Proceedings of the 40th ASMS Conference on Mass Spectrometry and Allied Topics; Washington, DC, 1992; $\mathrm{p} 10$.

4. Boyle, J. G.; Whitehouse, C. M. Anal. Chem. 1992, 64, 20842089.

5. Sin, C. H.; Lee, E. D.; Lee, M. L. Anal. Chem. 1991, 63, $2897-2900$.

6. Chien, B. M.; Michael, S. M.; Lubman, D.M. Anal. Chem. 1993, 65, 1916-1924.

7. Houk, R. S. Anal. Chem. 1986, 58, 97A-105A.

8. Myers, D. P.; Hieftje, G. M. Microchem. J. 1993, 48, 259-277.

9. Kutscher, R.; Grix, R.; Li, G.; Wollnik, H. Int. I. Mass Spectrom. Lon Processes 1991, 103, 117-128.

10. Green, L. W.; Macdonald, R. G.; Sopchyshyn, F. C. Anal. Instrum. 1988, 17, 195-214.

11. Fassett, J. D.; Walker, R. J.; Travis, J. C.; Ruegg, F. C. Int. J. Mass Spectrom. Ion Processes 1987, 75, 111-126.

12. Simmons, D. S. Int. I. Mass Spectrom. Ion Processes 1984, 55, 15-30.

13. Wiley, W. C.; McClaren, I. H. Rev. Sci. Instrum. 1955, 26, 1150-1157.

14. Fassel, V. A.; Bear, B. R. Spectrochim. Acta 1986, 41B, 10891113.

15. deHeer, W. A.; Milani, P. Rev. Sci. Instrum. 1991, 62, 670-677. 